\title{
Einleitung: \\ Reproduktionstechnologien, Generativität, Verwandtschaft
}

Als vor über vierzig Jahren - am 25. Juli 1978 - Louise Brown als das erste im Labor gezeugte Kind in der Nähe von Manchester zur Welt kam, wurde diese Geburt vom Informationsdienst der britischen Regierung aufgezeichnet und weltweit als Sensation gefeiert. Heute ist das Geburtsvideo auf Youtube allgemein zugänglich und die verschiedenen Formen assistierter Reproduktion sind zu einem etablierten Bestandteil der Fortpflanzungsroutinen geworden. Dazwischen liegen kontinuierliche Weiterentwicklungen der gen-, bio- und reproduktionstechnologischen Forschung und deren Institutionalisierung als Verfahren der künstlichen Befruchtung in sogenannten Kinderwunsch-Kliniken, in Samenbanken, Kryo-Konservierung von Eizellen, Vermittlungsagenturen für Eizell-Spenderinnen und Leihmütter, Pränataldiagnostik, vorgeburtliche Bluttests, Genetische Beratungsstellen und Datenbanken von Spenderkindern, die allesamt dafür gesorgt haben, dass die Reproduktionstechnologien zu einem Markt mit reger Nachfrage und zu einem Wirtschaftsfaktor wurden. Dazwischen liegen gleichfalls diverse gesellschaftspolitische Auseinandersetzungen und mediale Debatten über den Schutz des Embryos, über neue Formen der Eugenik und Bevölkerungspolitik, Designer-Babies, ethisch begründete Grenzen des Wunschs nach einem (gesunden) Kind, rechtliche Verbote wie auch gesetzliche Reglements neuer Verantwortlichkeiten angesichts vervielfältigter Familienverhältnisse sowie die in den 1980er und zu Beginn der 1990er Jahren breit geführte Debatte innerhalb der Frauenbewegung und ihre feministische Kritik der Reproduktionstechnologien (z. B. Corea 1985).

Fast in Vergessenheit geraten ist, wie außerordentlich breit und grundsätzlich politisch die feministische Kritik an den Gen- und Reproduktionstechnologien in ihren Anfängen ausgerichtet war. 1985 fand der erste Kongress "Frauen gegen Gentechnik und Reproduktionstechnik» in Bonn statt, der sich gegen die "Ausbeutung von Natur, Frauen und Dritte[r] Welt« (Die Grünen/AK Frauenpolitik 1986, 15) als »eine neue Form von Gewalt» gegen Frauen richtete, wie es im Aufruf zum Kongress hieß. Die Themenschwerpunkte und die Ausrichtung der Kritik folgten überwiegend einer differenztheoretischen und ökofeministischen Argumentation, 
in der die Unterdrückung von Frauen und die Ausbeutung von Natur im Zusammenhang wahrgenommen und verstanden wurden. Diese Perspektive dominierte auch in der internationalen feministischen Vernetzung gegen Gen- und Reproduktionstechniken FINRRAGE, die wichtige Impulse für die Analyse und den Widerstand in Deutschland setzte. Der zweite Kongress "Frauen gegen Gen- und Reproduktionstechnologien", der 1988 in Frankfurt am Main abgehalten wurde (Bradish et al. 1989), war davon geprägt, dass zwischenzeitlich die Bundesanwaltschaft Reproduktionstechnologien und Humangenetik zu »anschlagsrelevanten Themen" erklärt hatte. Bereits im Vorfeld des ersten Kongresses 1985 verübte die militante Frauengruppe Rote Zora einen Anschlag auf den im Bau befindlichen Technologiepark in Heidelberg und im August 1986 brachen Aktivistinnen der Roten Zora in das Humangenetische Institut in Münster ein. 1987 wurden Privatwohnungen mehrerer Aktivistinnen durchsucht, Ulla Penselin und Ingrid Strobl wegen "Unterstützung einer terroristischen Vereinigung" nach $\$ 129$ a festgenommen und mehrere Monate lang in Isolationshaft festgehalten. Der Frankfurter Kongress verabschiedete eine Solidaritätserklärung für die noch immer inhaftierte Ingrid Strobl, die von einem breiten Bündnis von Wissenschaftler_innen, Frauen- und Lesbengruppen, Behindertenverbänden, Gewerkschafts- und Kirchengruppen getragen wurde.

Inhaltlich kreisten die Debatten auf beiden Kongressen um den Begriff der Selbstbestimmung. Während Shulamith Firestone noch prognostiziert hatte, die Reproduktionstechnologien besäßen das Potenzial, die Frauen vom Joch ihrer Körpernatur und der Mutterschaft zu befreien, wie überhaupt mit der "künstlichen Fortpflanzung" die "Tyrannei der biologischen Familie [...] zerschlagen« werde $(1975,17)$, kritisierte Maria Mies den Begriff der Selbstbestimmung als bürgerliches Konstrukt, das den Kopf vom Körper trenne und damit unweigerlich zu einer Auslagerung der Reproduktionstechnologien an weibliche Andere in den Ländern des Südens führen werde, wie es heute Analysen zur globalisierten Ausbreitung der Reproduktionsmedizin bzw. des Reproduktionsmarktes zeigen (Bergmann 2014). Mies stellte heraus, dass das bürgerliche Besitzdenken des Kopfes über den Bauch es erlaube, Selbstbestimmung so zu denken, wie es in den jeweiligen Lebensplan passe (Mies 1986, 118). Zudem wurde der Begriff der Selbstbestimmung auch von Seiten behinderter Frauen kritisiert und der Einwand formuliert, dass er im Zusammenhang der mit den Reproduktionstechnologien verbundenen Pränataldiagnostik ein "soziales und individuelles Bewußtsein in Richtung auf technischen Machbarkeitswahn" (Köbsell / Waldschmidt 1989, 106) befördere. 
In den die Kongresse begleitenden Debatten wurde immer wieder die Befürchtung artikuliert, dass die neuen Reproduktionstechnologien die Selbstbestimmung von Frauen nur scheinbar erhöhten, sie faktisch hingegen einschränkten. Da die Entscheidungen von Frauen zur Nutzung der technologischen Möglichkeiten sich weitgehend in der Logik der technischen Machbarkeit bewegten, könne auch das der Technik eingeschriebene patriarchale Ziel - der Zugriff auf Frauenkörper und die weibliche Reproduktionsfähigkeit und deren Verwertbarkeit im Rahmen kapitalistischer Akkumulation - nicht umgangen oder vermieden werden (kritisch dazu: Treusch-Dieter 1990). Gleichermaßen problematisiert wurden nachfolgend das Recht und die Möglichkeiten der Selbstbestimmung über den eigenen Körper im Hinblick auf die Frage nach der Nutzung und dem Umgang mit den Angeboten der Pränataldiagnostik. Strittig waren die Rolle von (genetischen) Beratungsstellen und die sich unter dem - als Dogma empfundenen - Präventionsangebot vollziehende Intensivierung vorgeburtlicher Kontrolle zum Schutze des Fötus. Es wurde eingewendet, dass die pränataldiagnostischen Beratungsstellen zwar offiziell mit dem Ziel beraten sollten, nur die von der Schwangeren selbst in Einklang mit ihren Wertüberzeugungen getroffene Entscheidung zu stärken, die Möglichkeiten der Pränataldiagnostik insgesamt jedoch die gesellschaftliche Tendenz der Vermeidung von Geburten behinderter Kinder intensivierten und damit Frauen moralisch unter Druck setzten (z. B. Samerski 2002; Katz-Rothman 2012).

Heute hingegen ist die Frage der Selbstbestimmung dahinter verschwunden, dass Frauen überwiegend als Akteurinnen der Reproduktionstechnologien in Erscheinung treten: als Personen, die Körpersubstanzen "spenden" und ihre Körper "verleihen" bzw. "ausleihen", als Kundinnen und Ratsuchende, die - wie Malaika Rödel (2015) herausgearbeitet hat im medialen Diskurs nicht länger als weibliches Subjekt mit unerfülltem Kinderwunsch beschrieben werden, sondern als Teil einer Paar-Konstellation, die das Projekt Kind aktiv betreibt. Mittlerweile ist breite Normalisierung zu konstatieren (Deutsches IVF-Register 2017) und die Entwicklungen von Genetik, Stammzellforschung und Reproduktionsmedizin haben die Nutzung der Reproduktionstechnologien - Kryokonservierung von Ei- und Samenzellen in Kombination mit In-vitro-Fertilisationstechnologien - in globalem Umfang vorangetrieben, wenngleich mit stark unterschiedlichen nationalen Regelungen, vor allem im Hinblick auf die sogenannten Leih- oder Tragemutterschaft. Gesellschaftspolitisch hat sich ein Klima der Akzeptanz durchgesetzt, in dem einerseits ethische Standards des 1990 eingeführten Embryonenschutzgesetzes relativiert 
(z. B. Deutscher Ethikrat 2016) und ein »Fortpflanzungsmedizingesetz für Deutschland« (z.B. Beier et al. 2017) gefordert werden, und andererseits an der staatlichen Kontrolle des ungeborenen Lebens in der Form des Abtreibungsverbots festgehalten wird, wie jüngst die im Februar 2019 getroffene parlamentarische Entscheidung zur Beibehaltung des $\$ 219$ a zeigt.

Wissenschaftliche Fragestellungen und Untersuchungen richten sich aktuell verstärkt auf die transformativen Prozesse innerhalb der reproduktiven und verwandtschaftlichen Praktiken und Verhältnisse (Distinction 2015) und es zeichnen sich unterschiedliche Perspektivierungen ab. Grundlegend lässt sich fragen, ob die Reproduktionstechnologien die gesellschaftlich herrschenden Vorstellungen von Familie und Verwandtschaft verändern und inwiefern die neuen reproduktionstechnologischen Tatbestände zu gewandelten Vorstellungen von Zugehörigkeit und Abstammung führen. Gleichermaßen offen ist, welche Praktiken diesen etwaigen neuen Vorstellungen folgen. In dem Maße, wie sich die biologisch-soziale Selbstverständlichkeit der heterosexuellen Kleinfamilie auflöst, und auch die Familie den Pfad "from nature to choice" beschreitet - also zunehmend zu einer Sache von Wahl, Entscheidung, Intentionalität und individueller Autonomie wird -, nimmt das doing family andere Formen an, und es gilt zu beschreiben, welcher kommunikativer, medialer und praktischer Möglichkeiten es sich nun bedient und welche neuen Habitualisierungen sich herausbilden.

Nicht zuletzt wird Verwandtschaft selbst durch die technologischen Optionen und Mittel als Konstruktion sichtbar. Die "Bionormativität» (Witt 2014) des klassisch-triadischen Familienmodells gibt sich mit ihrer Doppelstruktur eben genau als Bio-Normativität zu erkennen, nämlich als ein Set natürlicher Tatsachen, die über Vereinbarungen, Recht und Moral reguliert werden. Die bürgerliche Kleinfamilie war eine kulturelle Form, die gleichwohl die Suggestion nähren konnte, in ihr kämen Natur und Kultur zur Deckung. Doch das ehemals fraglos als Natur Vorausgesetzte rückt nun selbst auf die Seite der Kultur, des Sozialen und Gesellschaftlichen. Die technologisch bedingte Entkoppelung von biologischer Verwandtschaft und Familienbildung dekonstruiert die Matrix >Heterosexualität - Generation - genetische Verbindung`, z. B. wenn es nun Vaterschaft auch ohne einen heterosexuellen Akt, ohne elterliche Ur-Szene gibt. Ob es sich freilich bewahrheitet, was jüngst - gleichsam als Widerhall der Firestone'schen Hoffnungen - zu lesen war, dass nämlich, "wenn es etwas gibt, das die Väterherrschaft beenden kann, [...] dies wahrscheinlich die schrittweise Verschiebung des Verständnisses von Geschlecht und Sexualität [ist]《 (Higgins 2018), bleibt abzuwarten. 
Es kann also weiter gehofft werden. Zwar sind die Reproduktionstechnologien aus dieser Perspektive nichts weniger als die ehedem perphorreszierten "tools of patriarchy", doch geraten sie gleichwohl als "tools of capitalism" ins Visier wissenschaftlicher Analysen. Neue Schnittstellen von Familie und Markt lassen sich ausmachen (Kitchen Politics 2015) und hier sind zwei Aspekte herauszustellen: Zum einen wurden reproduktionsmedizinische Vorgänge und generativ-reproduktive Arbeiten und Ressourcen zu einem Bestandteil der Wertproduktion und damit kommodifiziert. Zum anderen werden, da es biotechnisch möglich ist, einzelne Momente und Funktionen des Reproduktionsprozesses voneinander zu trennen und vertraglich zu regeln, auf diese Weise nicht nur die ehemals stabilen Bezugspunkte der heteronormativen Familie - Vaterschaft, Mutterschaft, Genealogie - auf tiefgreifende Weise infrage stellt, sondern verwandtschaftsbegründende Beziehungen auf eine neuartige Weise rechtlich regelungsbedürftig. Das Beispiel der Leihmutterschaft zeigt in besonderem Maße, dass neue Justierungen des Vertrags- und Familienrechts erforderlich sind. Die Etablierung eines vollentwickelten transnationalen Marktes für reproduktive Dienstleistungen - Dienstleistungen, die zur Reproduktion einer Familie beitragen ohne indes zur Familie zu sgehören - erfordert die Aufhebung der Rechtsgültigkeit biologischer Verwandtschaftsverhältnisse. Dies ist besonders bei der Leih- oder Tragemutterschaft der Fall, wo gegen das herkömmliche Verständnis von Mutterschaft, die an Schwangerschaft und Geburt gebunden ist, die Rechte der "intentionalen" Familie (intended parents) durchgesetzt werden.

Die mittlerweile auch disziplinär weit verzweigte Diskussion zur assistierten Reproduktion und ihren Konsequenzen kann im vorliegenden Heft nur punktuell eingefangen werden; mit den hier versammelten Beiträgen werden Schlaglichter geworfen, die sich um die Thematiken \Leihmutterschaft und $>$ Kulturelle Bilder und Konzepte von Verwandtschaft und Reproduktions(technologien) < gruppieren. Den zentralen Fokus bildet nicht zufällig die Leih- oder Tragemutterschaft. Wird bei dieser Form von Auslagerung der Mutterschaft zumeist aus einer globalisierungskritischen Perspektive die reproduktionsmedizinische Kolonialisierung von weiblichen Körpern und der Fruchtbarkeit von Frauen thematisiert, richten demgegenüber Ulrike Kadi und Katharina Leithner-Dziubas in ihrem Beitrag Das Monster einer zweibeinigen Gebärmutter. Leihmutterschaft als Ortswechsel den Blick nicht auf die realen Verhältnisse, sondern auf das Gefürchtete, auf unbewusste Phantasmen und Bilder, die einen abgewehrten monströsen Charakter der Leihmutterschaft artikulieren. Die "Territorialität« der Mutterschaft (Bernard), der Ur-Ort der Mutter hat sich zu Ort- 
schaften vervielfältigt. Diese innerpsychische Bühne, die immer auch von Spuren der Angst durchkreuzt ist, verknüpfen die Autorinnen mit Margret Atwoods Report der Magd, einem Werk, das bereits 1985 dieser Monstrosität in literarischer Fiktion Ausdruck verliehen und jüngst in der erfolgreichen Serienverfilmung ein breites Publikum erreicht hat.

Gleichfalls an ein öffentliches Publikum richtet sich die Theaterbühne, auf deren Brettern seit 1989 regelmäßig Auftragswerke lehrstückartig gesellschaftliche Reflexionen und Debatten zur Reproduktionsmedizin anstoßen. Birte Giesler führt mit Inszenierungen des Doing Family in einer biotechnisierten Kultur: Reproduktionstechnologien, Generativität und Verwandtschaft im >Biowissenschaftsdramar seit Rolf Hochhuths, Unbefleckte Empfängnisく die Leser_innen durch drei Beispiele dieser Gattung und spürt dem dort vorund aufgeführten »doing family« nach. Sie zeigt, dass auch die hier inszenierten neuen Praktiken von Reifizierungen der Geschlechterstereotypen durchzogen sind und als von individuellen Konsumwünschen Einzelner angetrieben erscheinen und damit kaum über die herkömmlich-bekannten prokreativen und generativen Strukturen hinausweisen, "wie auch immer diese konkret körper-leiblich gelebt werden mögen".

Genau für diese konkreten körperlichen Erlebnisse und leiblichen Erfahrungen interessiert sich Helga Krüger-Kirn, die dem schwangeren Leib als dem randeren Schauplatz $\prec$ Rechnung tragen will. Ihr Aufsatz Somatisches Wissen artikulieren. Annäherungen an die leiblichen Erfahrungen von Schwangerschaft und von Leihmutterschaft zielt auf eine Dimension, die sowohl in der quantifizierend operierenden medizinischen Betreuung wie auch in den kontroversen Diskussion über die Leihmutterschaft zu verschwinden droht: Das subjektive, leibliche Erleben von Schwangerschaft und eine Artikulation von Körpererfahrungen, die durchlässig ist für Imaginäres, für Ängste, Wünsche und Körperselbstbilder. Das Erleben »leiblicher Intersubjektivität« in der Schwangerschaft als ein Verhältnis zwischen dem somatisch Eigenen und Fremden nimmt in einer durchlebten Leihmutterschaft veränderte Züge an.

Der selbsterlebten Schwangerschaft steht die in Auftrag gegebene Schwangerschaft gegenüber: Julia Teschlade zeigt mit ihrem Beitrag Wenn das liebe Geld nicht wär': Zur Konstruktion von Intimität zwischen Tragemüttern und gleichgeschlechtlichen Männerpaaren von einer empirischen Studie ausgehend, welche Herausforderungen eine kommerzialisierte generative Intimität sowohl an die Auftraggeber wie an die Anbieterin stellt. Standen im klassischen Ehe- und Familienmodell reproduktive Dienstleistungen und reproduktive Arbeit - im Begriff Liebe verkapselt - in keinem direkten Bezug zum männlichen Familienlohn, so geraten nun Mutterschaft und 
Entlohnung in ein unmittelbares Verhältnis. Beobachten lässt sich, dass die Herstellung neuartiger intimer Verhältnisse zwischen einem homosexuellen Auftraggeber-Paar und der Tragemutter abermals durch die Abblendung der ökonomischen Dimension erfolgt und sich vorrangig in Formen von Fürsorglichkeit und Achtsamkeit artikuliert. Dennoch: Männer kaufen die Körperdienste einer Frau für ein eigenes Kind; in dieser Offenkundigkeit liegt auch neuer Legitimationsbedarf. Und in dem Maße, wie sich neuartige Konstellationen von Liebe und Geld zu etablieren beginnen, werden die alten Konstellationen reflexiv, nämlich ihrer Selbstverständlichkeit entkleidet und als Ordnungsmuster allererst sichtbar.

Die Reproduktionstechnologien bewirken somit nicht zuletzt eine nachhaltige Entplausibilisierung von lebensweltlichen Selbstverständlichkeiten. Dies zeigt auch Christina von Braun, indem sie in ihrem Beitrag Geist, Geld und Genealogie. Reproduktion und väterliche Blutslinie die Geschichte der Blutsverwandtschaft als ein Konstrukt der westlichen Welt nachzeichnet. Dabei wird deutlich, dass der referentielle Status, der dem natürlichen roten Blut zukommt, das durch die patrilinearen Stammbäume fließt, sich letztlich einem anderen Saft verdankt: der Tinte in den Formen von Schrift und Geld. Seit der Vater mit dem genetischen Fingerabdruck geworden ist, was er zuvor nie war, nämlich `certusı, identifizierbar, wird umso deutlicher, dass >der Vater nie eine biologische Kategorie dargestellt hatte, sondern geistig gedacht wurde und werden musste.

Der von Christina von Braun geschlagene weite historische Bogen veranschaulicht die Verschiebungen von Verwandtschaft auf der diachronen Ebene, korrespondierend dazu macht Marilyn Strathern den Blick aus der Ferne (auf den patriarchal-kapitalistischen Westen) zum Verständnis der durch die Reproduktionstechnologien initiierten Veränderungen auf der synchronen Ebene stark. In ihrem Beitrag Tauschverhältnisse und Konsumverhältnisse. Der Embryo als zukünftiger Konsument zeigt die Sozial- und Kulturanthropologin in Kontrastierung mit Geschlechter- und Verwandtschaftskonzepten aus Papua Neuguinea, wie sehr die gesellschaftlichen Auseinandersetzungen um Reproduktionstechnologien von grundlegenden Konzepten und Vorstellungen von Verwandtschaft, Generativität und personalem Geschlecht geprägt sind, in welche die technischen Neuerungen eingebettet sind und werden. Dementsprechend versteht Strathern die Reproduktionstechnologien nicht so sehr als Motoren von Dynamik und Veränderung, sondern beschreibt sie vielmehr als Produkt und Ergebnis der westlichen Gegenwartskultur und Gegenwartsgesellschaft, deren Kontinuität, Hartnäckigkeit und Immanenz sie durch systematische Reflexivität aufzubrechen bestrebt ist. Kleinschrittig und mit großer Prä- 
zision für scheinbar nebensächliche Details illustriert sie die Bandbreite und Formenvielfalt der neuen kulturellen Verwicklungen von KörperMaterie, Technik und Diskurs im Prozess der Verbreitung von Reproduktionstechnologien. Stratherns Beitrag wurde für dieses Heft aus dem Englischen übersetzt, dem Text vorangestellt ist eine Einführung in das relationale Denken von Strathern, die Michi Knecht und Katharina Liebsch verfasst haben. Sie führen in ihrem Beitrag Beziehungen sichtbar machen Debatten erweitern. Reproduktionstechnologien denken mit Marilyn Strathern in die Arbeitsweise der Autorin ein, lokalisieren den zur Übersetzung ausgewählten Text in seinen gesellschaftlichen, disziplinären und methodologischen Kontexten und arbeiten die Produktivität der Strathernschen Perspektivierungen für die analytische Durchdringung des Verhältnisses von Reproduktionstechnologien, Verwandtschaft und Geschlecht heraus. Stratherns Werben für eine kontinuierliche Arbeit an Begriffen und Konzepten unter systematischer Berücksichtigung empirisch dokumentierter Pluralität macht auch für die feministischen Diskussionen über die vielfältigen Praxen und Phänomene im Kontext der Reproduktionstechnologien deutlich, dass Theoriearbeit nicht durch politische Überzeugungen ersetzt werden kann.

Die Rubrik Im Gespräch ist dieses Mal doppelt bestückt. Thematisch einschlägig mit Blick auf den Schwerpunkt des Heftes ist das Gespräch zwischen Élisabeth Roudinesco, der bekannten französischen Historikerin der Psychoanalyse, und Manuela Fraire, der Vorsitzenden der römischen psychoanalytischen Vereinigung, das im Januar 2018 in Rom geführt wurde. Fraire knüpft dabei an Roudinescos Dialog mit Jacques Derrida Woraus wird Morgen gemacht sein? von 2001 an, der u.a. die Vielfalt der "neuen Familien« zum Thema hatte und diskutiert mit Roudinesco die Praxis der Leihmutterschaft.

Einen thematisch anderen Akzent setzen die Frankfurter Politikwissenschaftlerinnen Uta Ruppert und Tanja Scheiterbauer in ihrem Gespräch mit der nigerianisch-britischen Feministin und Professorin für Gender Studies an der University of California Davis, Amina Mama. Sie diskutieren Einflüsse und Strömungen des transnationalen Diskurses über »Feminisms in Africa im Spannungsfeld verschiedener Kulturen, Identitäten und Subjektivitäten und kommen auch auf die sozialpolitischen Verhältnisse von Gewalt, Militarismus und Geschlecht als Gegenstand der Forschungen von Amima Mama zu sprechen.

Der Essay Das Geschlecht vor dem Recht vor der Sprache steht in mehrfacher Hinsicht Außer der Reihe. Brigitte Rauschenbach hat uns damit kluge Überlegungen zum Gleichstellungsgebot der Verfassung und zur Bio-Lo- 
gik, zu Gleichheitsforderungen und Identitätspolitik sowie zur "Sprache des Geschlechts" hinterlassen. Ihr Anknüpfungspunkt ist die rechtliche Sanktion einer "Ehe für alle« und die rechtliche Anerkennung geschlechtlicher Vielfalt im Personenstandsgesetz. Der Essay unterhält vielfältige Bezüge zum Schwerpunkt des Heftes. Brigitte Rauschenbach hat das Inkrafttreten des Gesetzes, wonach Menschen mit einem »dritten Geschlecht" im Geburtenregister neben "männlich" und "weiblich" jetzt auch den Eintrag "divers« auswählen können, nicht mehr erlebt. Sie ist im Alter von 75 Jahren am 14. Dezember 2018 gestorben. Wir wünschen, dass ihrer in der lebendigen Auseinandersetzung mit dem letzten von ihr selbst fertig gestellten Text gedacht werde!

In der Rubrik Bilder und Zeichen präsentieren wir eine Photostrecke mit Arbeiten von Alexandra Bircken, die z. T. auch auf der diesjährigen Biennale in Venedig zu sehen sein werden. Ausgangspunkt des skulpturalen Schaffens von Bircken ist der menschliche Körper. Für unser Heft hat sie Arbeiten zusammengestellt, die die Themen Sexualität und Reprodution umkreisen. Auch das Titelbild stammt von der Künstlerin.

In eigener Sache gratulieren wir mit einer Collage von Mechthild Veil und einem Text von Ulla Wischermann unserer ehemaligen Mitherausgeberin Ute Gerhard zum 80. Geburtstag.

In der Rubrik Berichte stellen Sarah Hoesch und Laura Anna Klein Eindrücke und Ergebnisse der Internationalen Konferenz Motherhood and the Law vor, die vom 13.-15. September 2018 an der Universität Hildesheim stattfand.

Zudem erscheinen auch in diesem Heft Rezensionen, von denen einige das Schwerpunktthema des Heftes berühren. Einschlägig dafür ist der Rezensionsessay von Friederike Kuster mit dem Titel Ödipus revisited. Er behandelt Beiträge, die unter Stichwort: Sozialisation und familiale Triade in der Ausgabe 2/2018 der Zeitschrift westend die Ergebnisse eines Workshops des Instituts für Sozialforschung dokumentieren. Dort waren soziologische und sozialpsychologische Perspektiven auf die ödipale Triade angesichts der aktuellen - nicht zuletzt reproduktionstechnologisch bedingten Transformationen der Ordnung von Geschlecht und Generation entfaltet worden. Darüber hinaus stellt Susanne Schultz kritische Perspektiven auf die Bevölkerungswissenschaft vor, die Michelle Murphy in ihrem Buch The Economization of Life dargelegt hat.

Wir wünschen eine lebendige Lektüre und hoffen, die weitere Debatte angeregt zu haben. 


\section{Literatur}

Beier, Henning M. et al. (2017): Ein Fortpflanzungsmedizingesetz für Deutschland. Leopoldina. Nationale Akademie der Wissenschaften Diskussion Nr. 13. Halle.

Bergmann, Sven (2014): Ausweichrouten der Reproduktion. Biomedizinische Mobilität und die Praxis der Eizellspende. Wiesbaden.

Bradish, Paula/Feyerabend, Erika/Winkler, Ute (Hrsg.) (1989): Frauen gegen Genund Reproduktionstechnologien. Beiträge vom 2. Bundesweiten Kongress Frankfurt, 28.-30.10. 1988. München.

Corea, Gena (1986 [1985]): MutterMaschine. Reproduktionstechnologien. Von der künstlichen Befruchtung zur künstlichen Gebärmutter. Berlin.

Deutscher Ethikrat (2016): Embryospende, Embryoadoption und elterliche Verantwortung, Stellungnahme. Online unter: www.ethikrat.org.

Deutsches IVF-Register 2017: Jahrbuch 2016 In: Sonderheft Reproduktionstechnologie und Endokrinologie 14. Jahrgang 2017.

Die Grünen im Bundestag/AK Frauenpolitik und Sozialwissenschaftliche Forschung und Praxis für Frauen (Hrsg.) (1986): Frauen gegen Gentechnik und Reproduktionstechnik. Dokumentation zum Kongress 1985. Köln.

Distinction. Skandinavian Journal of Social Theory 16 (3) (2015): Themenheft: Reproduktion.

Firestone, Shulamith (1975): Frauenbefreiung und sexuelle Revolution. Frankfurt a. M.

Higgins, Charlotte (2018): The Guardian. In: der Freitag vom 24.12.2018.

Kitchen Politics (Hrsg.) (2015): Sie nennen es Leben, wir nennen es Arbeit. Biotechnologie, Reproduktion und Familie im 21. Jahrhunderts. Münster.

Köbsell, Swantje/Waldschmidt, Anne (1989): Pränatale Diagnostik, Behinderung und Angst. In: Bradish, Paula/Feyerabend, Erika/Winkler, Ute (Hrsg.): Frauen gegen Gen- und Reproduktionstechnologien. Beiträge vom 2. Bundesweiten Kongress Frankfurt, 28.-30.10.1988. München, 102-107.

Mies, Maria (1986): Indische Frauen zwischen Unterdrückung und Befreiung. Frankfurt a. M. Nahman, Michal (2013): Extractions. An Ethnography of Reproductive Tourism. Basingstoke.

Rödel, Malaika (2015): Geschlecht im Zeitalter der Reproduktionstechnologien. Natur, Technologie und Körper im Diskurs der Präimplantationsdiagnostik. Bielefeld.

Samerski, Silja (2002): Die verrechnete Hoffnung. Von der selbstbestimmten Entscheidung durch genetische Beratung. Münster.

Treusch-Dieter, Gerburg (1990): Von der sexuellen Rebellion zur Gen- und Reproduktionstechnologie. Tübingen.

Witt, Charlotte (2014): Family, Self and Society. A Critique of the Bionormative Conception of the Family. In: MacLeod, Carolyn/Baylis, Francois (Hrsg.): Family-Making. Contemporary Ethical Challenges. Oxford, 49-63. 